\title{
Topology Optimization in Hyperthermia Therapy
}

\author{
Alan A. S. Amad, Abimael F. D. Loula, Antonio A. Novotny \\ Laboratório Nacional de Computação Científica LNCC/MCTI, \\ Coordenação de Matemática Aplicada e Computacional, \\ Av. Getúlio Vargas 333, 25651-075 Petrópolis - RJ, Brasil \\ E-mail: alanasa@lncc.br, aloc@lncc.br, novotny@lncc.br
}

\begin{abstract}
Hyperthermia therapy is a medical treatment in which body tissue is exposed to slightly higher temperatures to damage and kill cancer cells or to make cancer cells more sensitive to the effects of radiation and certain anti-cancer drugs. To selectively heat the cancer region, antennas are positioned around of patient, emitting electromagnetic waves. One of the challenges in thermal therapy is delivering the appropriate amount of heat to the correct part of the patient's body. In this work, we develop a new optimization method, using topological derivative, which finds the best distribution of heat sources generated by antennas aiming at increasing the temperature in the region of the tumor. Numerical results are presented illustrating possible application of the proposed methodology.
\end{abstract}

Keywords: Cancer Treatment, Topological Derivatives, Electromagnetic Waves, Finite Element Method, Hyperthermia therapy

\section{Topological derivative concept}

The topological derivative measures the sensitivity of a given shape functional with respect to an infinitesimal singular domain perturbation, such as the insertion of holes, inclusions, sourceterms or even cracks. More precisely, the basic idea is to introduce a singular perturbation in the domain $\Omega$ confined in a small ball $B_{\varepsilon}(\widehat{x})$ of size $\varepsilon$ and center at $\widehat{x} \in \Omega$, as shown in fig. 1 , here $\widehat{x}$ is an arbitrary point of $\Omega$ and $\omega$ is a fixed bounded domain of $\mathbb{R}^{d}$. One can introduce a characteristic function associated to the unperturbed domain, $\chi=\mathbb{1}_{\Omega}$. Then, we define a characteristic function associated to the topologically perturbed domain of the form $\chi_{\varepsilon}$. In this work, $\chi_{\varepsilon}(\widehat{x})=\mathbb{1}_{\Omega}-(1-\gamma) \mathbb{1}_{\overline{B_{\varepsilon}(\widehat{x})}}$, where $\gamma \in \mathbb{R}$. Thus, a piecewise constant function $\gamma_{\varepsilon}$ can be defined as

$$
\gamma_{\varepsilon}=\gamma_{\varepsilon}(x):= \begin{cases}1 & \text { if } x \in \Omega \backslash \bar{B}_{\varepsilon} \\ \gamma & \text { if } x \in B_{\varepsilon}\end{cases}
$$

Then, assume that a given shape functional $\psi\left(\chi_{\varepsilon}(\widehat{x})\right)$, associated to the topologically perturbed domain, admits the following topological asymptotic expansion

$$
\psi\left(\chi_{\varepsilon}(\widehat{x})\right)=\psi(\chi)+\rho(\varepsilon) D_{T} \psi(\widehat{x})+o(\rho(\varepsilon)),
$$

where $\psi(\chi)$ is the shape functional associated to the original (unperturbed) domain, $\rho(\varepsilon)$ is a positive function such that $\rho(\varepsilon) \rightarrow 0$, when $\varepsilon \rightarrow 0$. The function $\widehat{x} \mapsto D_{T} \psi(\widehat{x})$ is called the topological derivative of $\psi$ at $\widehat{x}$. Therefore, this derivative can be seen as a first order correction of $\psi(\chi)$ to approximate $\psi\left(\chi_{\varepsilon}(\widehat{x})\right)$.

We adopt the methodology developed in [1], which is given by the following result [2]:

Theorem 1. Let $\psi\left(\chi_{\varepsilon}(\widehat{x})\right)$ be a shape functional associated to the topologically perturbed domain, which admits, for to $\varepsilon$ sufficiently small, the topological asymptotic expansion given by (2). 
Furthermore, it is assumed that the remainder $\mathcal{R}(\rho(\varepsilon))=o(\rho(\varepsilon))$ has the following additional property $\mathcal{R}^{\prime}(\rho(\varepsilon)) \rightarrow 0$, when $\varepsilon \rightarrow 0$. Therefore, the topological derivative can be written as

$$
D_{T} \psi(\widehat{x})=\lim _{\varepsilon \rightarrow 0} \frac{1}{\rho^{\prime}(\varepsilon)} \frac{d}{d \varepsilon} \psi\left(\chi_{\varepsilon}(\widehat{x})\right),
$$

where $\frac{d}{d \varepsilon} \psi\left(\chi_{\varepsilon}(\widehat{x})\right)$ is the derivative of $\psi\left(\chi_{\varepsilon}(\widehat{x})\right)$ with respect to the small parameter $\varepsilon$.

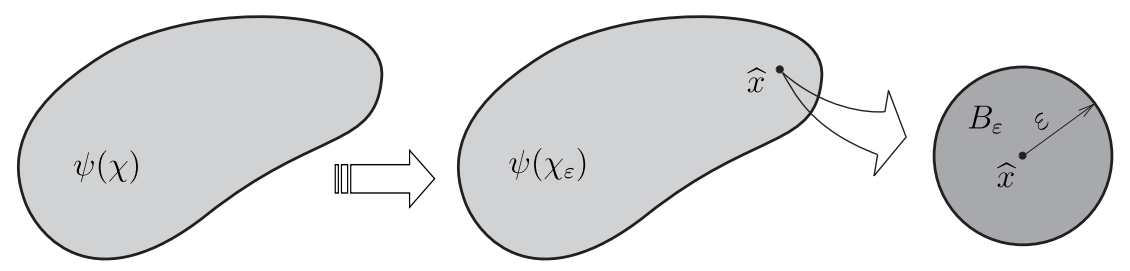

Figura 1: The topological derivative concept.

\section{Model problem}

Let $\Omega \subset \mathbb{R}^{d}(d \geq 2)$ be an open and bounded domain with a Lipschitz continuous boundary $\partial \Omega$. Let $\mathcal{B}$ be a subset of $\Omega(\mathcal{B} \subset \Omega)$ with $\partial \mathcal{B}$ used to denote the boundary of $\mathcal{B}$, as shown in figure 2. Our goal is to maximized the temperature in the cancer domain $\mathcal{D} \subset \mathcal{B}$, by keeping the temperature in the healthy region $\mathcal{B} \backslash \mathcal{D}$.

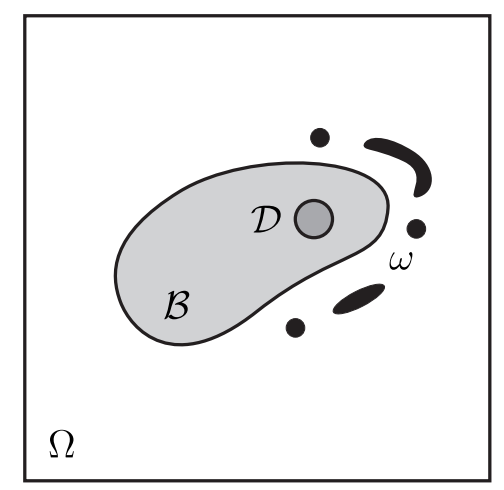

Figura 2: Generic representation of the domain $\Omega$, where $\omega$ are antennas that emit electromagnetic waves in all domain $\Omega$ passing through the healthy body $\mathcal{B}$ such that the diseased tissue (cancer) $\mathcal{D}$ is heated.

\subsection{Unperturbed problem}

According to the subject matter hereof, the following shape functional is adopted

$$
\psi(\chi):=\mathcal{J}(\theta)=-\frac{w}{|\mathcal{D}|} \int_{\mathcal{D}} \theta+\frac{1-w}{|\mathcal{B}|} \int_{\mathcal{B} \backslash \overline{\mathcal{D}}} \theta
$$

where $0<w<1$ and $\theta$ is the temperature distribution, solution to the following problem

$$
P_{\theta}:\left\{\begin{array}{l}
\text { Find } \theta \in H_{0}^{1}(\Omega), \text { such that } \\
\int_{\Omega}(\alpha \nabla \theta \cdot \nabla \eta+\beta \theta \eta)=\int_{\Omega} \frac{\sigma|u|^{2}}{2} \eta, \quad \forall \eta \in H_{0}^{1}(\Omega),
\end{array}\right.
$$


where $|u|^{2}=u \bar{u}$, with $\bar{u}$ denotes the complex conjugate of $u$.

In addition $u$ is solution to the following time-harmonic wave problem in weak form

$$
P_{u}:\left\{\begin{array}{l}
\text { Find } u \in \mathbf{H}^{1}(\Omega), \text { such that } \\
\int_{\Omega}\left(\nabla u \cdot \nabla \bar{\eta}-k^{2} u \bar{\eta}\right)+\boldsymbol{i} k \int_{\partial \Omega} u \bar{\eta}=\int_{\Omega} f \bar{\eta}+\int_{\partial \Omega} r \bar{\eta}, \quad \forall \eta \in \mathbf{H}^{1}(\Omega)
\end{array}\right.
$$

We define the complex Hilbert space $\mathbf{L}^{2}(\Omega):=\left\{v: \Omega \rightarrow \mathbb{C}, \int_{\Omega}|v|^{2} d \Omega<\infty\right\}$ of squareintegrable functions, equipped with the inner product $(v, w)=\int_{\Omega} v \bar{w} d \Omega$, and the norm $\|$ $v \|_{\mathbf{L}^{2}(\Omega)}=(v, v)^{1 / 2}$. And define the subspace $\mathbf{H}^{1}(\Omega):=\left\{v \in \mathbf{L}^{2}(\Omega): \nabla v \in \mathbf{L}^{2}(\Omega)\right\}$ where $\nabla v$ are the weak derivatives of the function $v$. The inner product $(v, w)_{1}=\int_{\Omega} \nabla v \cdot \nabla \bar{w} d \Omega$ induces the norm $\|v\|_{\mathbf{H}^{1}(\Omega)}=(v, v)_{1}^{1 / 2}$.

For the classical Hilbert space we define $L^{2}(\Omega):=\left\{v: \Omega \rightarrow \mathbb{R}, \int_{\Omega}|v|^{2} d \Omega<\infty\right\}$ of square-integrable functions, equipped with the classical inner product. And define the subspace $H^{1}(\Omega):=\left\{v \in L^{2}(\Omega): \nabla v \in L^{2}(\Omega)\right\}$, where $\nabla v$ are the weak derivatives of the function $v$.

The problem presented in (6) is the wead form at Helmholtz problem with Robin boundary condition. Problem (5) is the weak form of a steady-state heat transfer problem with homogeneous Dirichlet boundary condition. By solving the Helmholtz problem (6), we obtain the solution $u$ to be replaced in (5), thus we find the temperature $\theta$ of the body $\mathcal{B}$ due to the antenna $\omega$, which radiates electromagnetic waves. Rewriting the problems given by (5) and (6), in theirs strong forms, we obtain the following semi-coupled system of PDE.

$$
H P\left\{\begin{aligned}
-\Delta u-k^{2} u & =f, & & \text { in } \Omega, \\
-\operatorname{div}(\alpha \nabla \theta)+\beta \theta & =\frac{\sigma|u|^{2}}{2}, & & \text { in } \Omega, \\
\frac{\partial u}{\partial \mathbf{n}}+\boldsymbol{i} k u & =r, & & \text { on } \partial \Omega, \\
\theta & =0, & & \text { on } \partial \Omega,
\end{aligned}\right.
$$

where $\boldsymbol{i}=\sqrt{-1}, k \in \mathbb{R}$ is the wavenumber, $\sigma$ is the electrical conductivity of biological material, $r \in H^{-\frac{1}{2}}(\partial \Omega), \alpha, \beta \in \mathbb{R}^{+}$and $f \in \mathbf{L}^{2}(\Omega)$ source term described as:

$$
f= \begin{cases}1, & \text { in } \omega \\ 0, & \text { in } \Omega \backslash \bar{\omega}\end{cases}
$$

where $\omega \subset \Omega \backslash \overline{\mathcal{B}}$ are antennas that emit electromagnetic waves. In addition

$$
\begin{gathered}
\alpha=\left\{\begin{array}{lll}
\alpha_{1}, & \text { in } \mathcal{B} \backslash \overline{\mathcal{D}}, \\
\alpha_{2}, & \text { in } \mathcal{D}, \\
\alpha_{0}, & \text { in } \Omega \backslash \overline{\mathcal{B}},
\end{array}\right. \\
\beta=\left\{\begin{array}{lll}
\beta_{1}, & \text { in } \mathcal{B} \backslash \overline{\mathcal{D}}, \\
\beta_{2}, & \text { in } \mathcal{D}, \\
\beta_{0}, & \text { in } \Omega \backslash \overline{\mathcal{B}},
\end{array}\right.
\end{gathered}
$$

with $\mathcal{B}$ the healthy body and $\mathcal{D}$ the cancer (figure 2 ).

To simplify our analysis, two adjoint problems are introduced. The first one is associated with the semi-coupled heat problem (5), that is

$$
\left\{\begin{array}{l}
\text { Find } \varphi \in H_{0}^{1}(\Omega), \text { such that } \\
\int_{\Omega}(\alpha \nabla \varphi \cdot \nabla \eta+\beta \varphi \eta)=-\frac{w}{|\mathcal{D}|} \int_{\mathcal{D}} \eta+\frac{1-w}{|\mathcal{B}|} \int_{\mathcal{B} \backslash \overline{\mathcal{D}}} \eta, \quad \forall \eta \in H_{0}^{1}(\Omega) .
\end{array}\right.
$$


The other one is associated with the Helmholtz problem (6), namely

$$
\left\{\begin{array}{l}
\text { Find } v \in \mathbf{H}^{1}(\Omega), \text { such that } \\
\int_{\Omega}\left(\nabla v \cdot \nabla \bar{\eta}-k^{2} v \bar{\eta}\right)+\boldsymbol{i} k \int_{\partial \Omega} v \bar{\eta}=\int_{\Omega} \sigma u \varphi \bar{\eta}, \quad \forall \eta \in \mathbf{H}^{1}(\Omega) .
\end{array}\right.
$$

Note that the problem (12) depends on the solutions to (6) and (11), while the problem (11) is independent of the others problems due to the choice of the shape function (4)

\subsection{Perturbed problem}

The aim of our work is to use the topological derivative to find the best design of the antenna that radiates electromagnetic waves in order to maximize the temperature in the region $\mathcal{D} \subset \mathcal{B}$ where the cancer is located. Then consider the perturbed shape functional

$$
\psi\left(\chi_{\varepsilon}\right):=\mathcal{J}\left(\theta_{\varepsilon}\right)=-\frac{w}{|\mathcal{D}|} \int_{\mathcal{D}} \theta_{\varepsilon}+\frac{1-w}{|\mathcal{B}|} \int_{\mathcal{B} \backslash \overline{\mathcal{D}}} \theta_{\varepsilon},
$$

where $0<w<1$ and $\theta_{\varepsilon}$ is the temperature distribution, solution of the (14).

$$
\left\{\begin{array}{l}
\text { Find } \theta_{\varepsilon} \in H_{0}^{1}(\Omega), \text { such that } \\
\int_{\Omega}\left(\alpha \nabla \theta_{\varepsilon} \cdot \nabla \eta+\beta \theta_{\varepsilon} \eta\right)=\int_{\Omega} \frac{\sigma\left|u_{\varepsilon}\right|^{2}}{2} \eta, \quad \forall \eta \in H_{0}^{1}(\Omega) .
\end{array}\right.
$$

In addition $u_{\varepsilon}$ is solution of (15).

$$
\left\{\begin{array}{l}
\text { Find } u_{\varepsilon} \in \mathbf{H}^{1}(\Omega), \text { such that } \\
\int_{\Omega}\left(\nabla u_{\varepsilon} \cdot \nabla \bar{\eta}-k^{2} u_{\varepsilon} \bar{\eta}\right)+\boldsymbol{i} k \int_{\partial \Omega} u_{\varepsilon} \bar{\eta}=\int_{\Omega} f_{\varepsilon} \bar{\eta}+\int_{\partial \Omega} r \bar{\eta}, \quad \forall \eta \in \mathbf{H}^{1}(\Omega) .
\end{array}\right.
$$

From (14) and (15), we write the perturbed hyperthermia problem, in strong form, as

$$
H P P\left\{\begin{aligned}
-\Delta u_{\varepsilon}-k^{2} u_{\varepsilon} & =f_{\varepsilon}, & & \text { in } \Omega \\
-\operatorname{div}\left(\alpha \nabla \theta_{\varepsilon}\right)+\beta \theta_{\varepsilon} & =\frac{\sigma\left|u_{\varepsilon}\right|^{2}}{2}, & & \text { in } \Omega \\
\frac{\partial u_{\varepsilon}}{\partial n}+u_{\varepsilon} & =r, & & \text { on } \partial \Omega \\
\theta_{\varepsilon} & =0, & & \text { on } \partial \Omega
\end{aligned}\right.
$$

where $r$ is the prescribed source term on $\partial \Omega$ and $f_{\varepsilon}$ the perturbed source term described as:

$$
\begin{gathered}
f_{\varepsilon}(x)=\left\{\begin{array}{rr}
\gamma(x), & x \in \omega_{\varepsilon}(\widehat{x}), \\
0, & x \notin \omega_{\varepsilon}(\widehat{x}),
\end{array}\right. \\
\omega_{\varepsilon}(\widehat{x})=\left\{\begin{array}{rr}
\omega \backslash B_{\varepsilon}(\widehat{x}), & \widehat{x} \in \omega, \\
\omega \cup B_{\varepsilon}(\widehat{x}), & \widehat{x} \notin \omega,
\end{array}\right.
\end{gathered}
$$

where $\omega_{\varepsilon} \subset \Omega \backslash \overline{\mathcal{B}}$ is the ball of radius $\varepsilon$ and $\gamma$ is given by:

$$
\gamma(x)=\left\{\begin{aligned}
1, & \text { if } \quad x \notin \omega \\
-1, & \text { if } \quad x \in \omega
\end{aligned}\right.
$$

Similarly to the problems (6) and (5), we introduce the adjoint-state associated to the perturbed problem (15):

$$
\left\{\begin{array}{l}
\text { Find } v_{\varepsilon} \in \mathbf{H}^{1}(\Omega), \text { such that } \\
\int_{\Omega}\left(\nabla v_{\varepsilon} \cdot \nabla \bar{\eta}-k^{2} v_{\varepsilon} \bar{\eta}\right)+\boldsymbol{i} k \int_{\partial \Omega} v_{\varepsilon} \bar{\eta}=\int_{\Omega} \sigma u_{\varepsilon} \varphi \bar{\eta}, \quad \forall \eta \in \mathbf{H}^{1}(\Omega) .
\end{array}\right.
$$




\subsection{Stability conditions}

The following lemmas, which will not be proved here, express the stability of the perturbed problem defined by $H P P$.

Lemma 2. Let be $\widetilde{u}_{\varepsilon}=u_{\varepsilon}-u$, where $u$ and $u_{\varepsilon}$ are solutions of the Eq. (6) and Eq. (15), respectively. Then, we have the following estimate $\left\|\widetilde{u}_{\varepsilon}\right\|_{H^{1}(\Omega)} \leq C \varepsilon$, where $C$ is a constant independent of the parameter $\varepsilon$.

Lemma 3. Let be $\widetilde{\theta}_{\varepsilon}=\theta_{\varepsilon}-\theta$, where $\theta$ and $\theta_{\varepsilon}$ are solutions of the Eq. (5) and Eq. (14), respectively. Then, we have the following estimate $\left\|\widetilde{\theta}_{\varepsilon}\right\|_{H^{1}(\Omega)} \leq C \varepsilon$, where $C$ is a constant independent of the parameter $\varepsilon$.

Lemma 4. Let be $\widetilde{v}_{\varepsilon}=v_{\varepsilon}-v$, where $v$ and $v_{\varepsilon}$ are solutions of the Eq. (12) and Eq. (20), respectively. Then, we have the following estimate $\left\|\widetilde{v}_{\varepsilon}\right\|_{H^{1}(\Omega)} \leq C \varepsilon$, where $C$ is a constant independent of the parameter $\varepsilon$.

\section{Calculation of topological derivative}

In this section we present the calculation for the topological derivative. As shown in Section 1, the topological sensitivity analysis provides asymptotic development (2) for a given shape functional, called topological derivative (3), which measures the sensitivity of this functional when an infinitesimal singular perturbation is introduced at an arbitrary point of the domain [2]. Thus, after deriving the functional (13) with respect to the small parameter $\varepsilon$ we have

$$
\dot{\psi}\left(\chi_{\varepsilon}\right)=-\frac{w}{|\mathcal{D}|} \int_{\mathcal{D}} \dot{\theta}_{\varepsilon}+\frac{1-w}{|\mathcal{B}|} \int_{\mathcal{B} \backslash \overline{\mathcal{D}}} \dot{\theta}_{\varepsilon},
$$

In the perturbed heat problem given by (14), we derive with respect the $\varepsilon$ that

$$
\int_{\Omega}\left(\alpha \nabla \dot{\theta}_{\varepsilon} \cdot \nabla \eta+\beta \dot{\theta}_{\varepsilon} \eta\right)=\Re\left(\int_{\Omega} \sigma u_{\varepsilon} \overline{\dot{u}}_{\varepsilon} \eta\right), \forall \eta \in H_{0}^{1}(\Omega) .
$$

The same we do with the Helmholtz perturbed problem (15)

$$
\int_{\Omega}\left(\nabla \dot{u}_{\varepsilon} \cdot \nabla \bar{\eta}-k^{2} \dot{u}_{\varepsilon} \bar{\eta}\right)+\boldsymbol{i} k \int_{\partial \Omega} \dot{u}_{\varepsilon} \bar{\eta}=\gamma \int_{\partial B_{\varepsilon}} \bar{\eta}, \quad \forall \eta \in \mathbf{H}^{1}(\Omega) .
$$

In the adjoint heat problem given by (11), we set $\eta=\dot{\theta}_{\varepsilon}$, then

$$
\int_{\Omega}\left(\alpha \nabla \varphi \cdot \nabla \dot{\theta}_{\varepsilon}+\beta \varphi \dot{\theta}_{\varepsilon}\right)=-\frac{w}{|\mathcal{D}|} \int_{\mathcal{D}} \dot{\theta}_{\varepsilon}+\frac{1-w}{|\mathcal{B}|} \int_{\mathcal{B} \backslash \overline{\mathcal{D}}} \dot{\theta}_{\varepsilon}
$$

In the $(22)$ we chose $\eta=\varphi$, thus

$$
\int_{\Omega}\left(\alpha \nabla \dot{\theta}_{\varepsilon} \cdot \nabla \varphi+\beta \dot{\theta}_{\varepsilon} \varphi\right)=\Re\left(\int_{\Omega} \sigma u_{\varepsilon} \overline{\dot{u}}_{\varepsilon} \varphi\right),
$$

where $\Re(u)$ denotes the real part of $u$.

The new relation of the shape functional is given through the (24) and (25)

$$
\dot{\psi}\left(\chi_{\varepsilon}\right)=\Re\left(\int_{\Omega} \sigma u_{\varepsilon} \overline{\dot{u}}_{\varepsilon} \varphi\right) .
$$

Now, in the adjoint Helmholtz problem (20)) we set $\eta=\dot{u}_{\varepsilon}$, thus, after lacking the real part of the obtained results, we have

$$
\Re\left(\int_{\Omega}\left(\nabla v_{\varepsilon} \cdot \nabla \overline{\dot{u}}_{\varepsilon}-k^{2} v_{\varepsilon} \overline{\dot{u}}_{\varepsilon}\right)+i k \int_{\partial \Omega} v_{\varepsilon} \overline{\dot{u}}\right)=\Re\left(\int_{\Omega} \sigma u_{\varepsilon} \varphi \overline{\dot{u}}_{\varepsilon}\right) .
$$


In equation (23) we have $\eta=v_{\varepsilon}$, yielding, after taking the real part of the obtained results

$$
\Re\left(\int_{\Omega}\left(\nabla \dot{u}_{\varepsilon} \cdot \nabla \bar{v}_{\varepsilon}-k^{2} \dot{u}_{\varepsilon} \bar{v}_{\varepsilon}\right)+i k \int_{\partial \Omega} \dot{u} \bar{v}_{\varepsilon}\right)=\gamma \Re\left(\int_{\partial B_{\varepsilon}} \bar{v}_{\varepsilon}\right) .
$$

Using the relation of the functional form (27) with the variational problem given by (28) and expanding in powers of $\varepsilon$, we obtain

$$
\dot{\psi}\left(\chi_{\varepsilon}\right)=2 \pi \varepsilon \gamma \Re(\bar{v}(\widehat{x}))+o(\varepsilon),
$$

where we have used Lemma 4 together with the interior elliptic regularity of function $v$. Observe that by expanding the (29) in powers of $\varepsilon$, we obtain the following result

Theorem 5. The topological derivative of the shape function (4) is given by

$$
D_{T} \psi(\widehat{x})=\gamma \Re(\bar{v}(\widehat{x}))=\left\{\begin{aligned}
\Re(\bar{v}(\widehat{x})), & \text { if } \widehat{x} \notin \omega, \\
-\Re(\bar{v}(\widehat{x})), & \text { if } \widehat{x} \in \omega .
\end{aligned}\right.
$$

Result of some numerical experiments are presented next.

\section{Numerical results}

To illustrate the use of the proposed optimization method numerical experiments are solved in domain, shown in figure 3 . The domain is defined in a quadrangular geometry $\Omega=(-1,1) \times$ $(-1,1)$, the healthy body $\mathcal{B}$ on a circle of radius $\rho_{\mathcal{B}}=0.2$, the diseased region of body $\mathcal{D}$ is also circular with radius radius $\rho_{\mathcal{D}}=0.04$ and the antennas $\omega$ will be positioned over an annular region, with radius varying from 0.3 to 0.6 , i.e., $0.3 \leq \rho_{\omega} \leq 0.6$.

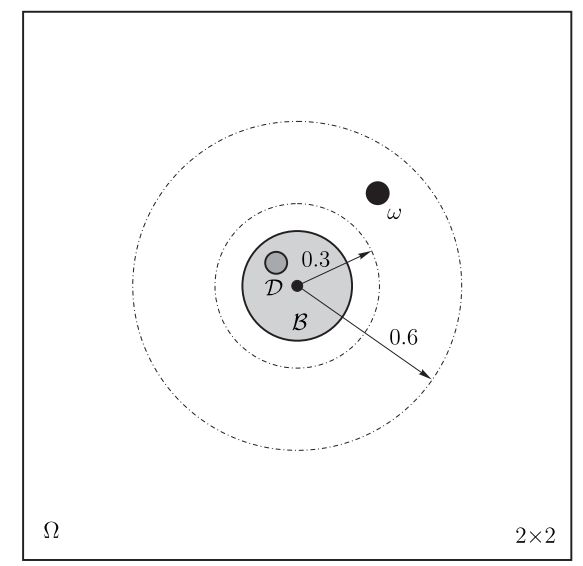

Figura 3: Domain of the experiments

In order to obtain a satisfactory solution of the problems (6) and (15) we need a mesh with elements of size $h$, which satisfy the following condition: $k^{2} h<1$, where $k$ is the wavenumber. Then, we used the classical Galerkin finite element method and uniform mesh with $10^{6}$ of linear triangular elements.

\subsection{Experiment - Choice of the wavenumber $k$ and initial guess of the $\omega$}

As initial experiment we positioned the target $(\mathcal{D})$ at the center o $\mathrm{ft}$ he body. The figure 4 (left), show the initial configuration of the $\omega$ and the distribution of heat on $\mathcal{B}$. The figure 4 (right), show the final configuration of the $\omega$ and the distribution of heat on $\mathcal{B}$.

In the following experiment we change the position of the target $\mathcal{D}$ at coordinates $(0.1,0.1)$, as shown in figure 5 . In left, show the initial configuration and in right, show the final configuration.

In figure 6 we show the decay of the cost function at each iteration. The optimal solution is found with only 11 iterations using a fixed point method. 

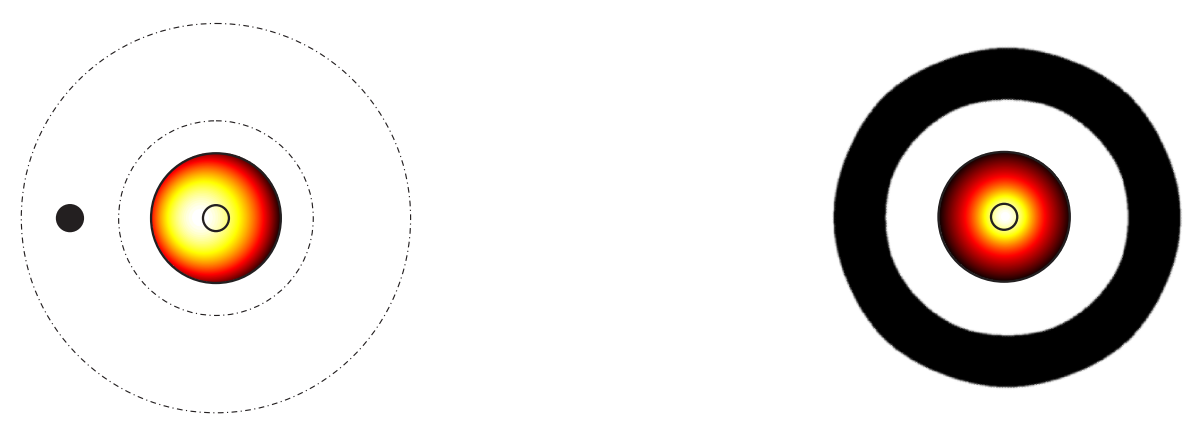

Figura 4: Experiment with the target at coordinates $(0.0,0.0)$ and $k=20$. configuration.
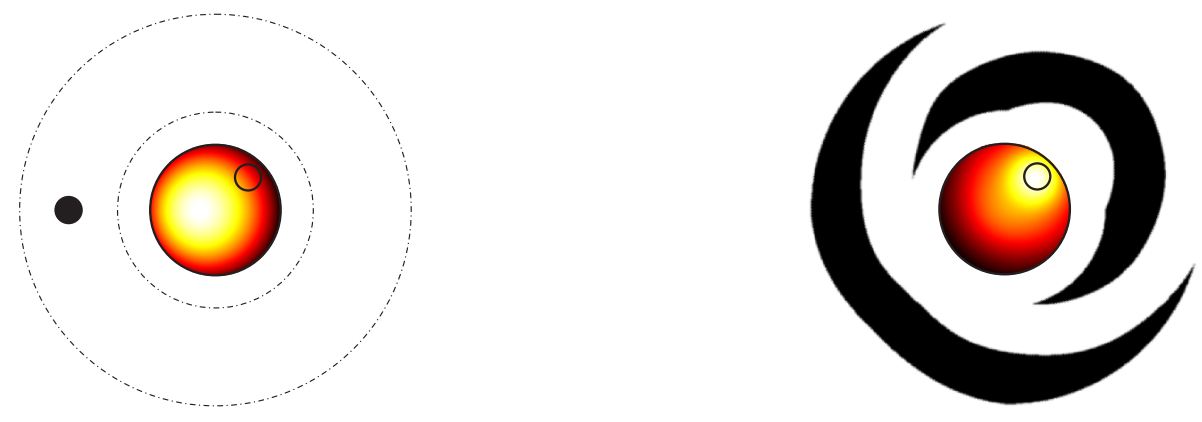

Figura 5: Experiment with the target at coordinates $(0.1,0.1)$ and $k=20$.

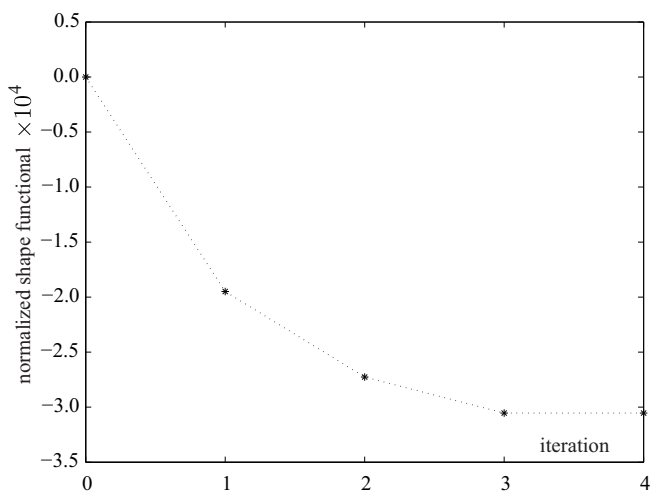

Figura 6: Evolution of the objective function in experiment with the target at coordinates $(0.1,0.1)$ and $k=20$.

\section{Conclusions}

We propose a new optimization method for the hyperthermia problem using topological derivatives. The preliminary work shows very promising results on heating selectively the region of the target.

\section{Referências}

[1] A. A. Novotny, Análise de sensibilidade topológica, Ph.D. Thesis, Laboratório Nacional de Computação Científica, Petrópolis, Brazil (2003).

[2] A. A. Novotny, J. Sokołowski, Topological derivatives in shape optimization, Interaction of Mechanics and Mathematics, Springer, 2013. 